\title{
Equivalent-Circuit Modeling of Lossless and Lossy Bi-Periodic Scatterers by an Eigenstate Approach
}

This paper was downloaded from TechRxiv (https://www.techrxiv.org).

LICENSE

CC BY-NC-SA 4.0

SUBMISSION DATE / POSTED DATE

$14-10-2021 / 18-10-2021$

\section{CITATION}

Hernández-Escobar, Alberto; Abdo-Sánchez, Elena; Esteban, Jaime; Martín-Guerrero, Teresa María; Camacho-Peñalosa, Carlos (2021): Equivalent-Circuit Modeling of Lossless and Lossy Bi-Periodic Scatterers by an Eigenstate Approach. TechRxiv. Preprint. https://doi.org/10.36227/techrxiv.16811092.v1

DOI

10.36227/techrxiv.16811092.v1 


\title{
Equivalent-Circuit Modeling of Lossless and Lossy Bi-Periodic Scatterers by an Eigenstate Approach
}

\author{
Alberto Hernández-Escobar, Jaime Esteban, Elena Abdo-Sánchez, Member, IEEE, \\ Teresa M. Martín-Guerrero, Member, IEEE, and Carlos Camacho-Peñalosa, Life Senior Member, IEEE
}

\begin{abstract}
The use of an eigenstate based equivalent circuit topology is proposed for the analysis and modeling of lossless and lossy bi-periodic scatterers. It can significantly simplify the design of this kind of surfaces, since it reduces the number of elements with respect to other general circuits. It contains at most only two admittances and two transformers depending on one unique transformation ratio. The real parts of these admittances can be assured to be non-negative, an interesting aspect in the modeling of lossy surfaces such as those present in asorbers. Moreover, due to the capability of decomposition into the eigenexcitations of the structure, the circuit provides important physical insight. Different cases of scatterers have been analyzed: symmetric and asymmetric, lossy and lossless. In all these cases, the modeling of the circuit admittances has been successfully achieved with a few RLC elements, positive and frequency independent. In the case of structures with symmetries, the transformation ratio directly reflects the physical orientation of the eigenexcitations of the scatterer. Furthermore, in the case of lossy scatterers but without symmetries, the resulting equivalent circuit reveals that their eigenexcitations are not linear polarizations, but elliptic polarizations whose properties are described by the complex transformation ratio.
\end{abstract}

Index Terms-Eigenexcitations, eigenstates, equivalent circuits, frequency-selective surfaces, lattice network, rasorber, reflectarray, scatterer, transmitarray.

\section{INTRODUCTION}

Nowadays, one of the usual ways to approach the analysis and synthesis of bi-periodic surfaces is by means of FEM or FDTD full-wave electromagnetic simulation [1]-[14]. However, the use of equivalent circuits is also common tool and is increasingly used [8]-[21]. The reasons for this are manifold. Equivalent circuits provide information about the behavior of the periodic surface in a more understandable and convenient way than the mere parameterization of simulation results. They allow more sophisticated multilayer structures to be analyzed and designed without resorting to repeated electromagnetic simulations, which are much more costly in terms of CPU

Manuscript received Month DD, YYYY; revised Month DD, YYYY; accepted Month DD, YYYY. This work was supported by the Spanish Ministerio de Ciencia, Innovación y Universidades (MCIU), the Agencia Estatal de Investigación (AEI) and the Fondo Europeo de Desarrollo Regiona (FEDER) (Programa Estatal de I+D+i Orientada a los Retos de la Sociedad) under grant RTI2018-097098-J-I00 and by the regional government (Junta de Andalucía) PAIDI 2020 under grant PY20_00452.

A. Hernández-Escobar, E. Abdo-Sánchez, T. M. Martín-Guerrero and C. Camacho-Peñalosa are with the Instituto Universitario de Investigación en Telecomunicación, Escuela Técnica Superior de Ingeniería de Telecomunicación, Universidad de Málaga, Andalucía Tech, 29010 Málaga, Spain (email: $\{$ ahe,elenaabdo,teresa,ccp\}@ic.uma.es).

J. Esteban is with the Information Processing and Telecommunications Center, Escuela Técnica Superior de Ingenieros de Telecomunicación, Universidad Politécnica de Madrid, 28040 Madrid, Spain (e-mail: jesteban@etc.upm.es). time. And they allow all existing knowledge of circuit theory to be used in the design of multilayer surfaces.

On the one hand, there are equivalent circuits that simply seek to model the results of the simulations [16], [17]. They can be used with any geometry, no matter how complicated it may be, because their starting point is the result of an electromagnetic simulation obtained by numerical methods. On the other hand, there are those that seek to provide information on the behavior of the unit cell by analytical methods, avoiding simulations [18], [19]. Although their subsequent use is much more efficient, their scope of application is limited to geometries which, although interesting, must be relatively simple, since they require simplifications and hypotheses on the fields in their slots or the currents in their conductors. But there is an additional option, which is to extract information from the computational effort of one or a few electromagnetic simulations, to obtain a circuit with a greater field of application than the simulated geometry from which it was obtained [20], [21].

In any case the choice of circuit topology is also relevant, since choosing one or another topology can facilitate or hinder the modeling. An unsuitable topology can lead to circuit elements with peculiar variations with frequency, or to negative resistances, capacitances and inductances. These may be correct and valid circuits, but their ability to explain the behavior of the periodic surface is lost and may complicate their use in design tasks.

This paper proposes the use of an eigenstate-based topology [22], [23], with several implications. The first one is the remarkable simplification of the extraction of the eigenexcitations of the unit cell (the crystal axes in the terminology of [21]). On the other hand, this topology leads to circuits in which it is ensured that their immittances have nonnegative real parts. The latter is particularly relevant, given the importance of absorbing surfaces [13]-[15], [24]-[28], which must obviously be modeled with lossy circuits and therefore with resistances (which had better be positive and constant). Additionally, the deduction of the eigenvectors of the circuit, associated to the eigenexcitations of the periodic surface, has led to find out the peculiar characteristics of these excitations in the case of cells that simultaneously show losses and lack of symmetries.

Section II of this article focuses on the basic ideas of the modeling of scatterers of negligible thickness by means of equivalent circuits, the particularization of the eigenstate circuit to these periodic surfaces, and its use. Section III explores a series of examples and how they particularize the 

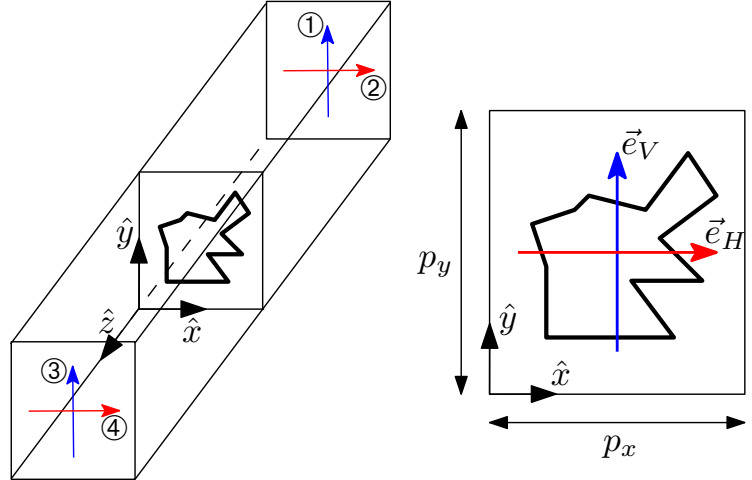

Fig. 1. Geometry of the unit cell of a bi-periodic surface and polarizations used in its analysis. The polygon shown as an example can represent either the metallization (high pass surface) or the slot (low pass).

proposed circuit, depending on whether 1) the scatterer (either lossy or lossless) has a symmetry plane, 2) has $90^{\circ}$ rotation symmetry, 3) is lossless with or without symmetry, or 4) has losses and no symmetry. The derivation of the eigenexcitations in the latter case is left for the Appendix, and the conclusions of this work are summarized in Section IV.

\section{Bi-PERIODIC SURFACE ANALYSiS}

The customary approach to the analysis of bi-periodic surfaces is the simulation of a unit cell (see Fig. 1), where a scatterer lays between walls of periodic boundary conditions. These boundary conditions depend on the excitation, i.e., the direction of the wave impinging on the surface. In our case we will consider normal incidence, $\theta^{i n c}=0$, and the usual decomposition into TE and TM polarizations. For the analysis, the $\varphi^{i n c}=0$ plane is chosen, so that a first polarization corresponds to the vertical electric field $\left(\vec{e}_{V}\right.$ along the $y$ axis) and a second polarization to the horizontal electric field ( $\vec{e}_{H}$ along $x$-axis). As is also usual, the thickness of the metallization is small enough to assume zero thickness.

If the frequencies to be considered do not exceed the cut-off frequency of the first higher-order Floquet mode, the scatterer behavior can be represented by a four-port circuit, as shown in Fig. 2(a). The normal incidence under any angle $\varphi$ can be described by a linear combination of the vertical and horizontal polarization incidences, or by the excitation of ports (1) and (2) of the circuit in Fig. 2(a), with the corresponding amplitude coefficients.

As explained in [29], the zero-thickness approximation implies the continuity of the electric field for each of the polarizations, so that the $S$-parameter matrix has the form:

$$
[S]=\left[\begin{array}{cccc}
S_{11} & S_{12} & 1+S_{11} & S_{12} \\
S_{12} & S_{22} & S_{12} & 1+S_{22} \\
1+S_{11} & S_{12} & S_{11} & S_{12} \\
S_{12} & 1+S_{22} & S_{12} & S_{22}
\end{array}\right]
$$

From these $S$-parameters, the impedance or the admittance matrix of the two-port in Fig. 2(b) can be obtained. Whereas in [29] the impedance matrix is used, here the use of the admittance matrix is preferred:

$$
\left[Y_{d}\right]=\left[\begin{array}{ll}
y_{11} & y_{12} \\
y_{12} & y_{22}
\end{array}\right]
$$

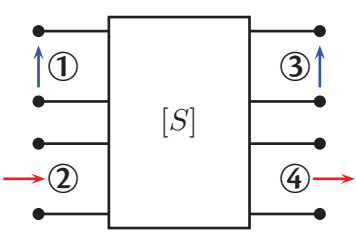

(a)

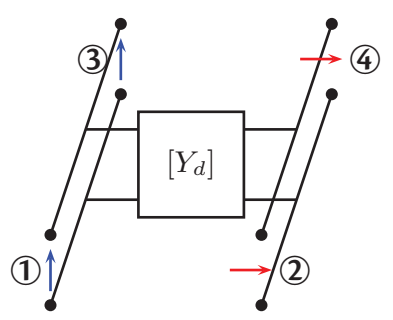

(b)
Fig. 2. (a) Circuit representation of a bi-periodic scatterer as four-port network, and ports definition. (b) Equivalent circuit by means of a two-port network.

whose elements can be written in terms of the $S$-parameters of the four-port as:

$$
\begin{aligned}
& y_{11}=\left(\frac{-2}{\eta_{0}}\right) \frac{\Delta S+S_{11}}{\Delta S+S_{11}+S_{22}+1} \\
& y_{12}=\left(\frac{-2}{\eta_{0}}\right) \frac{S_{12}}{\Delta S+S_{11}+S_{22}+1} \\
& y_{22}=\left(\frac{-2}{\eta_{0}}\right) \frac{\Delta S+S_{22}}{\Delta S+S_{11}+S_{22}+1}
\end{aligned}
$$

being $\Delta S=S_{11} S_{22}-S_{12}^{2}$.

\section{A. Eigenstate Circuit Topology}

As for any other (lossy or lossless) reciprocal circuit the two-port in Fig. 2(b) can be represented by the topology proposed in [22], which is based on the eigenstate decomposition. To get the most out of this topology it is necessary to reformulate the proposal in [22] by means of a simple change of variable, but with important implications. Let us consider $p=\tan \phi$ and rewrite the complex parameter $p$ in [22, (4)] as

$$
p=\frac{y_{11}-y_{22}}{2 y_{12}}+\sqrt{\left(\frac{y_{11}-y_{22}}{2 y_{12}}\right)^{2}+1} .
$$

Squaring this value leads to

$$
p^{2}=1+2\left(\frac{y_{11}-y_{22}}{2 y_{12}}\right) p .
$$

Then,

$$
\tan 2 \phi=\frac{2 \tan \phi}{1-\tan ^{2} \phi}=\frac{2 p}{1-p^{2}}=\frac{2 y_{12}}{y_{22}-y_{11}} .
$$

Now, using (3), the complex angle $\phi$ can be directly written in terms of the $S$-parameters of the unit cell:

$$
\tan 2 \phi=\frac{2 S_{12}}{S_{22}-S_{11}}
$$

or

$$
\phi=\frac{1}{2} \arctan \left(\frac{2 S_{12}}{S_{22}-S_{11}}\right) \pm k \frac{\pi}{2},
$$

where $k$ is an arbitrary integer whose only effect is to interchange the order of the eigenvectors and their corresponding eigenvalues. 


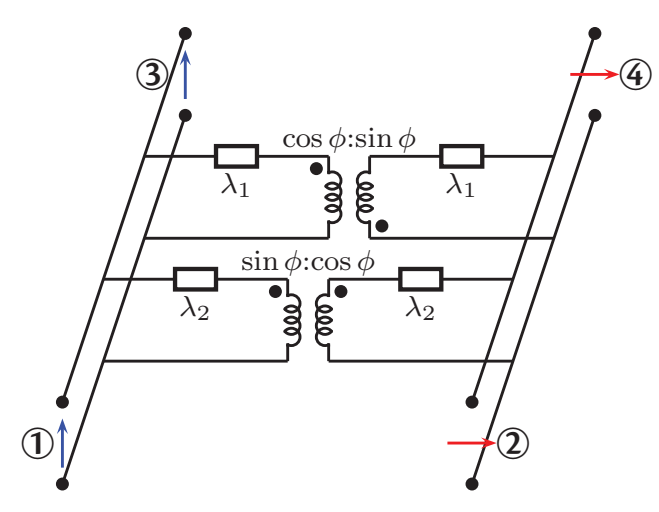

Fig. 3. Equivalent circuit for vertical and horizontal polarizations of a biperiodic scatterer based on eigenstates.

The change of variable also has effects on the eigenvectors of the circuit which become

$$
\vec{v}_{1}=\left[\begin{array}{c}
\sin \phi \\
\cos \phi
\end{array}\right] \quad ; \quad \vec{v}_{2}=\left[\begin{array}{c}
-\cos \phi \\
\sin \phi
\end{array}\right] .
$$

Obviously these two vectors will be unitary if $\phi$ is real.

The eigenvalues are expressed as

$$
\lambda_{1}=y_{22}+y_{12} \tan \phi \quad ; \quad \lambda_{2}=y_{11}-y_{12} \tan \phi,
$$

or, in terms of the $S$-parameters, as

$$
\begin{aligned}
& \lambda_{1}=\left(\frac{-2}{\eta_{0}}\right) \frac{\Delta S+S_{22}+S_{12} \tan \phi}{\Delta S+S_{11}+S_{22}+1} \\
& \lambda_{2}=\left(\frac{-2}{\eta_{0}}\right) \frac{\Delta S+S_{11}-S_{12} \tan \phi}{\Delta S+S_{11}+S_{22}+1}
\end{aligned}
$$

Finally, the transformers of the circuit proposed in [22], with turn ratios 1:p and $p: 1$, can be replaced by transformers of ratios $1: \tan \phi$ and $\tan \phi: 1$, or else, by transformers of ratios $\cos \phi: \sin \phi$ and $\sin \phi: \cos \phi$, respectively. The equivalent circuit for the four-port in Fig. 2(b) is then the one shown in Fig. 3, which makes use of two transformers and two different admittances: $\lambda_{1}$ and $\lambda_{2}$.

It is worth noting that the eigenvectors of the equivalent circuit are, directly, what we could refer to as 'eigenexcitations' of the scatterer or unit cell. If the eigenexcitation corresponding to $\vec{v}_{1}$ is considered:

$$
\vec{E}_{1}^{i n c}=\sin \phi \vec{e}_{V}+\cos \phi \vec{e}_{H}
$$

the reflected and transmitted fields are obtained by means of (1) as

$$
\begin{aligned}
\vec{E}_{1}^{r e f}= & \left(S_{11} \sin \phi+S_{12} \cos \phi\right) \vec{e}_{V} \\
& +\left(S_{12} \sin \phi+S_{22} \cos \phi\right) \vec{e}_{H} \\
\vec{E}_{1}^{t r m}= & \left(\left(1+S_{11}\right) \sin \phi+S_{12} \cos \phi\right) \vec{e}_{V} \\
& +\left(S_{12} \sin \phi+\left(1+S_{22}\right) \cos \phi\right) \vec{e}_{H}
\end{aligned}
$$

whereas the eigenexcitation corresponding to $\vec{v}_{2}$ is:

$$
\vec{E}_{2}^{i n c}=-\cos \phi \vec{e}_{V}+\sin \phi \vec{e}_{H} .
$$

Using (7) it is straightforward to prove that

$$
\begin{aligned}
& \vec{E}_{1}^{i n c} \times \vec{E}_{1}^{r e f}=0 \\
& \vec{E}_{1}^{i n c} \times \vec{E}_{1}^{t r m}=0 \\
& \vec{E}_{2}^{i n c} \cdot \vec{E}_{1}^{r e f}=0 \\
& \vec{E}_{2}^{i n c} \cdot \vec{E}_{1}^{t r m}=0 .
\end{aligned}
$$

That is, the incidence with one of the eigenexcitations only generates reflected and transmitted fields proportional to that excitation and no field proportional to the other eigenexcitation. Undoubtedly, this is a similar result to that of the diagonalization of the scattering matrix described in [21]. However, the use of the circuit herein proposed obtains the directions of the eigenexcitations in a much simpler way, and solves certain problem not addressed in [21], as will be discussed later in Section III-E.

\section{B. Use of the Equivalent Circuit}

One of the important advantages of the proposed circuit is that the admittances it includes always have non-negative real parts, as was proved in [22]. Therefore, it is expected that it will be possible to model both admittances by means of positive RLC components.

Estimates of the admittances and their variation in frequency, $\widehat{\lambda_{1}}$ and $\widehat{\lambda_{2}}$, can be obtained, and modeled with RLC elements, from the electromagnetic simulation of the unit cell (or, when available, by analytical methods). From these estimates it is straightforward to obtain the $S$ parameters of the scatterer as:

$$
\begin{aligned}
& \widehat{S_{11}}=-\frac{\widehat{\lambda_{1}} \eta_{0}}{2+\widehat{\lambda_{1}} \eta_{0}} \sin ^{2} \widehat{\phi}-\frac{\widehat{\lambda_{2}} \eta_{0}}{2+\widehat{\lambda_{2}} \eta_{0}} \cos ^{2} \widehat{\phi} \\
& \widehat{S_{12}}=\frac{\eta_{0}\left(\widehat{\lambda_{2}}-\widehat{\lambda_{1}}\right)}{\left(2+\widehat{\lambda_{1}} \eta_{0}\right)\left(2+\widehat{\lambda_{2}} \eta_{0}\right)} \sin 2 \widehat{\phi} \\
& \widehat{S_{22}}=-\frac{\widehat{\lambda_{1}} \eta_{0}}{2+\widehat{\lambda_{1}} \eta_{0}} \cos ^{2} \widehat{\phi}-\frac{\widehat{\lambda_{2}} \eta_{0}}{2+\widehat{\lambda_{2}} \eta_{0}} \sin ^{2} \widehat{\phi}
\end{aligned}
$$

where $\widehat{\phi}$ can be an estimation or a simple model of the variation with frequency of $\phi$, when such variation occurs.

\section{DisCUSSION AND APPLICATION EXAMPLES}

The behavior of the unit cell depends on its symmetries and loss characteristics. The proposed equivalent circuit reflects these symmetries and characteristics, and leads to certain particular configurations that are worth commenting on.

\section{A. Unit Cell With a Symmetry Plane}

In the case of a scatterer with a plane of symmetry (with or without losses) it is obvious that there are two linear polarizations, perpendicular to each other, which constitute the eigenexcitations of the unit cell, namely, the polarization along the plane of symmetry and the one perpendicular to it: $\vec{e}_{1}$ and $\vec{e}_{2}$, as shown in Fig. 4(a). If these polarizations were used for the analysis of the scatterer, $S_{12}=0$ would be obtained. Equation (8) then gives a value of $\phi=0$, and the transformers, which in that case would be of 1:0 and 0:1 ratios, degenerate 


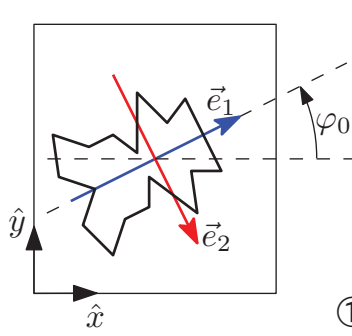

(a)

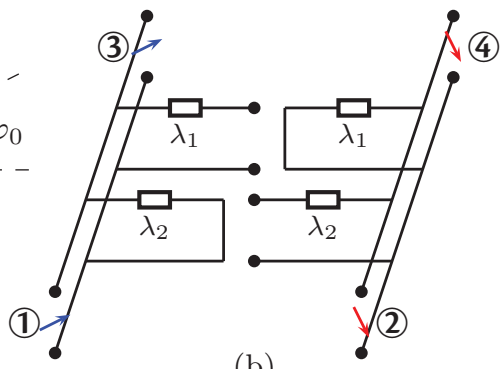

(b)

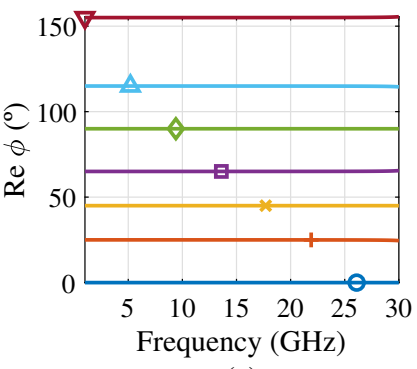

(a)

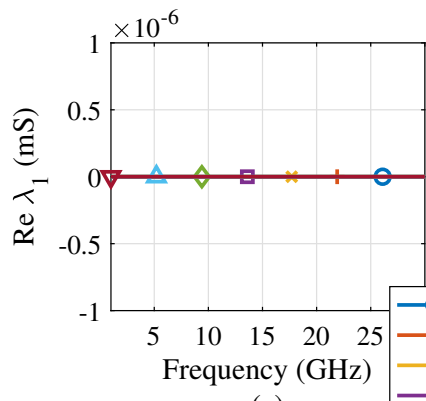

(c)

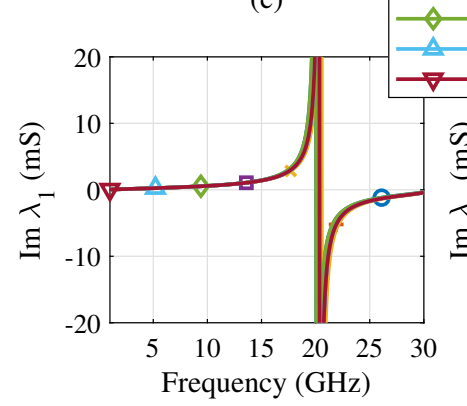

(e)

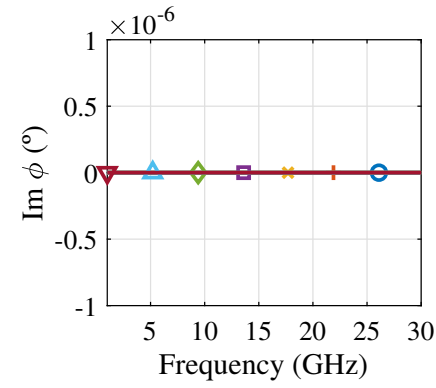

(b)
Fig. 4. (a) Scatterer with a symmetry plane. (b) Equivalent circuit for polarizations along, and perpendicular to, its symmetry axis.

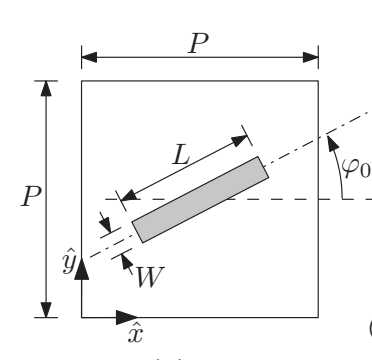

(a)

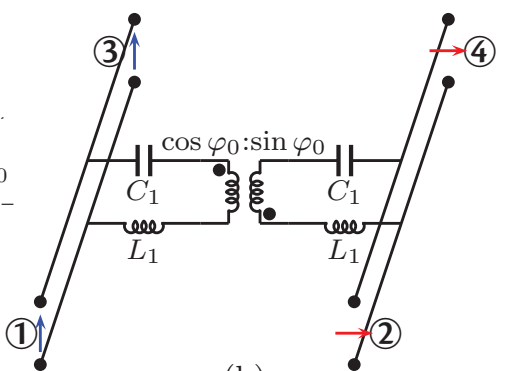

(b)
Fig. 5. (a) Bi-periodic rotated dipole in [29, Sec. III.B]. (b) Equivalent circuit for vertical and horizontal polarizations when $P=10 \mathrm{~mm}, L=7 \mathrm{~mm}$, $W=0.5 \mathrm{~mm}$, and for any angle $\varphi_{0}$. The values of the circuit components are $L_{1}=9.1 \mathrm{nH}$ and $C_{1}=6.8 \mathrm{fF}$.

into open and short circuits. The resulting circuit is shown in Fig. 4(b), where it is apparent that each of the admittances $\lambda_{1}$ and $\lambda_{2}$ is the independent and specific response of the unit cell to each of its eigenexcitations.

When vertical and horizontal polarizations are used for the analysis, the more general circuit of Fig. 3 should contain the same admittances $\lambda_{1}$ and $\lambda_{2}$. The role of the transformers will be the change of basis from the $\left(\vec{e}_{V}, \vec{e}_{H}\right)$ pair of Fig. 1(b) to the $\left(\vec{e}_{1}, \vec{e}_{2}\right)$ pair of Fig. 4(a) through the real angle $\varphi_{0}$.

A scatterer composed of a simple dipole can serve as an example. Let us consider the rotated dipole analyzed in [29, Sec. III.B] with $P=10 \mathrm{~mm}, W=0.5 \mathrm{~mm}, L=7 \mathrm{~mm}$ and different values of $\varphi_{0}$ (related with the parameter $\alpha$ in [29] as $\left.\alpha=\pi / 2-\varphi_{0}\right)$. Its shape is reproduced in Fig. 5(a). The results of the extraction of the equivalent circuit of Fig. 3 from HFSS simulations of the unit cell are shown in Fig. 6 for a number of values of $\varphi_{0}$. The figures illustrate the ability of the circuit to correctly detect the positioning of the dipole $(\phi=$ $\left.\varphi_{0}\right)$, as well as the invariance of the admittances $\lambda_{1}$ and $\lambda_{2}$ with angle $\varphi_{0}$. The real parts of the admittances are negligible, as corresponds to a lossless scatterer, and the imaginary part of $\phi$ is also zero, as expected.

In [29], the electromagnetic simulation of a whole series of dipole angular positions was necessary to obtain a large set of equivalent-circuit element values. However, in our case (like in [21]), a single analysis is necessary at one of the angular positions to obtain a circuit valid for any angle $\phi=\varphi_{0}$, and with unique admittances that can be approximated with Sec. III.B] for different rotation angles $\varphi_{0}$, simulated with HFSS. (a) and (b) angle $\phi$. (c) and (e) admittance $\lambda_{1}$. (d) and (f) admittance $\lambda_{2}$.

positive parameters as:

$$
\begin{aligned}
\widehat{\lambda_{1}} & =\frac{1}{j \omega L_{1}+\frac{1}{j \omega C_{1}}} \\
\widehat{\lambda_{2}} & =j \omega C_{2}
\end{aligned}
$$

with $L_{1}=9.1 \mathrm{nH}, C_{1}=6.8 \mathrm{fF}$ and $C_{2}=0.15 \mathrm{fF}$.

Moreover, for this unit cell composed of a thin dipole, the small capacitance $C_{2}$ is negligible, and thus also the effect of admittance $\lambda_{2}$. The equivalent circuit is then reduced to the one shown in Fig. 5(b), and the unit-cell $S$-parameters obtained with it are compared in Fig. 7 with the electromagnetic simulation and with the T-network circuit proposed in [29]. As can be seen, the agreement over the entire bandwidth of the fundamental mode is as good as that obtained in [29], but with a simpler circuit, obtained with less computational effort, and without the need for parameterization with the $\varphi_{0}$ angle.

It should be noted that, somehow, the symmetry of the unitcell is also lost by the interaction with its neighbors. This is evident when the size of the scatterer is such that it approaches that of the cell period, $P$. Repeating the analysis for a larger dipole $(L=8 \mathrm{~mm}, W=4 \mathrm{~mm})$ yields the results shown in Fig. 8. It can be seen that the angle $\phi$ no longer exactly
Fig. 6. Parameters of the equivalent circuit of the dipole proposed in [29, 


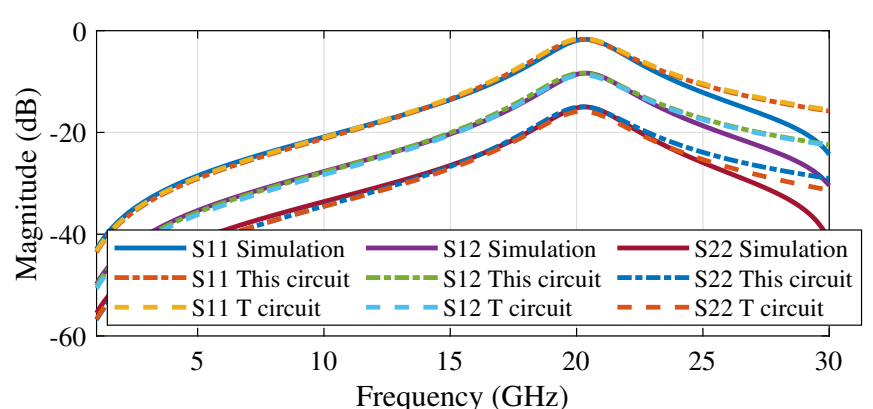

(a)

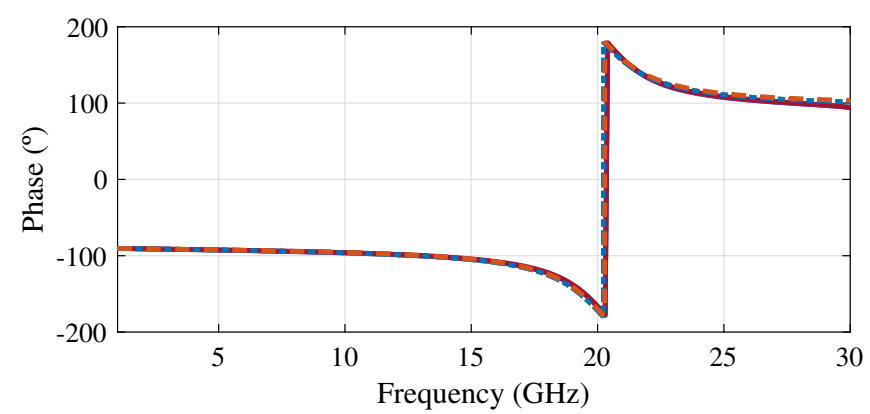

(b)

Fig. 7. S-parameters of the unit-cell formed by a dipole rotated $25^{\circ}$, obtained from simulation results, from the proposed equivalent circuit, and from the $\mathrm{T}$ circuit proposed in [29, Sec. III.B]. (a) Magnitude. (b) Phase.

agrees with $\varphi_{0}$, and that the admittances vary slightly with the angular position. Fig. 8(a) shows how, for rotations of $0^{\circ}$ and $45^{\circ}$, the angle obtained does correspond to the physical position of the dipole at any frequency. This is because, for these two angles, the periodic structure as a whole maintains the symmetry of the unit cell, which is not the case for intermediate angles. Anyway, as proposed in [21], it is possible to model the variation of $\phi$ with $\varphi_{0}$ and the frequency, as well as to parametrize the circuit elements of the admittances with $\phi$. In all cases, these will be smooth variations of easy modeling.

\section{B. Lossy Scatterer With a Symmetry Plane}

As already mentioned, one of the advantages of the proposed circuit worth noting is that it guarantees admittances with non-negative real parts. Consider as an example the resistively loaded dipole antenna proposed in [25, Fig. 3] and reproduced in the inset of Fig. 9(d). The effect of the supporting dielectric is not negligible in this case. Therefore, the whole unit cell with the dipole antenna on its dielectric substrate has been simulated with HFSS. To obtain the $S$ parameters of the scatterer, the effect of the dielectric is deembedded by simply changing the reference impedance at ports (3) and (4), shifting the reference planes by the length corresponding to the substrate thickness, and returning to the original reference impedances (vacuum impedance) at all four ports.

The result, considering the symmetry of the dipole with respect to the vertical plane, leads to $S_{12}=0$, and therefore $\phi=0$. Both polarizations are isolated from each other, and the only relevant parameters are the admittances $\lambda_{1}$ and $\lambda_{2}$,

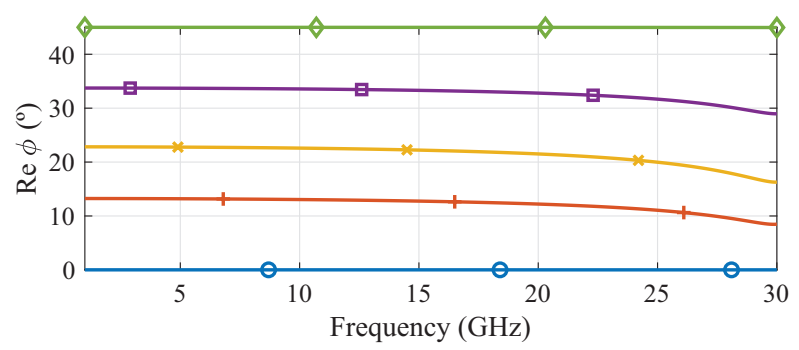

(a)

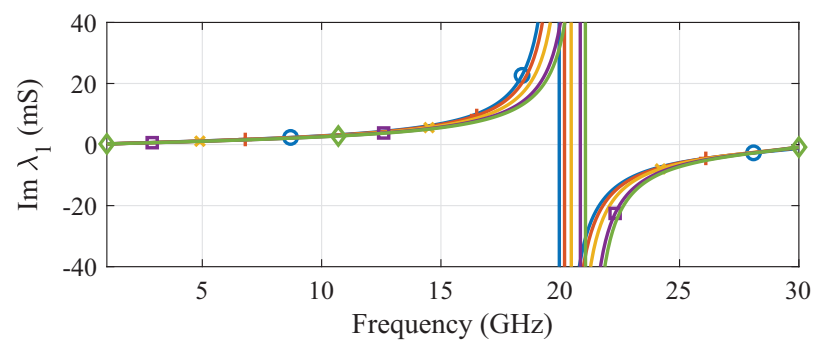

(b)

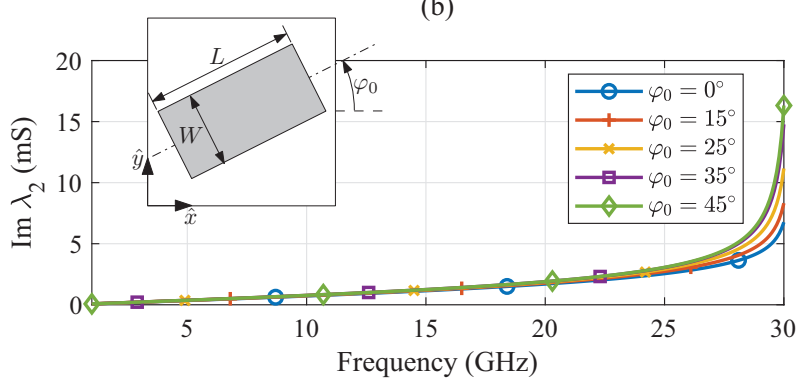

(c)

Fig. 8. Equivalent circuit parameters of the equivalent circuit for a larger dipole $(L=8 \mathrm{~mm}$ and $W=4 \mathrm{~mm})$ and for different rotation angles $\varphi_{0}$, from simulations with HFSS. (a) Angle $\phi$. (b) Admittance $\lambda_{1}$. (c) Admittance $\lambda_{2}$.

whose real and imaginary parts are shown in Fig. 9. A good approximation of these admittances is obtained by means of lossy series resonators:

$$
\begin{aligned}
\widehat{\lambda_{1}} & =\frac{1}{R_{1}+j \omega L_{1}+\frac{1}{j \omega C_{1}}}+\frac{1}{j \omega L_{2}+\frac{1}{j \omega C_{2}}} \\
\widehat{\lambda_{2}} & =\frac{1}{R_{3}+j \omega L_{3}+\frac{1}{j \omega C_{3}}}
\end{aligned}
$$

with $R_{1}=227 \Omega, L_{1}=5.53 \mathrm{nH}, C_{1}=107 \mathrm{fF}, L_{2}=3.9 \mathrm{nH}$, $C_{2}=13.6 \mathrm{fF}, R_{3}=3.07 \Omega, L_{3}=1.64 \mathrm{nH}$ and $C_{3}=$ $40 \mathrm{fF}$. All of them are positive values, and correctly reproduce the behavior of the admittances (see Fig. 9) except for the anomalous peak at $11.2 \mathrm{GHz}$. This peak is caused by a Fano resonance, which could also be modeled, although it would require the use of two coupled RLC resonators [30], what is beyond the scope of this paper.

Using this equivalent circuit and incorporating the supporting dielectric and ground plate, the response of the absorber designed in [25] can be reproduced. The Fig. 10 shows the comparison between the complete electromagnetic simulation of the structure and the results obtained using the proposed 


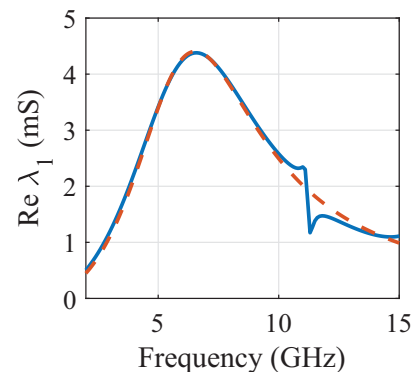

(a)

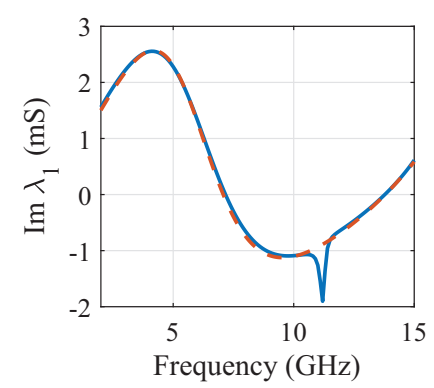

(c)

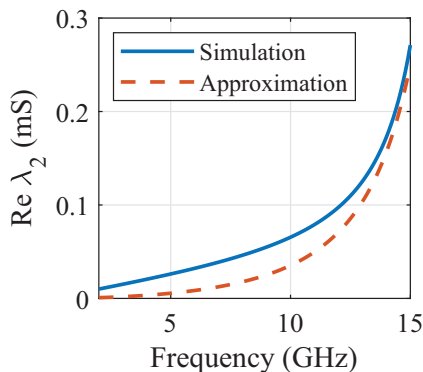

(b)

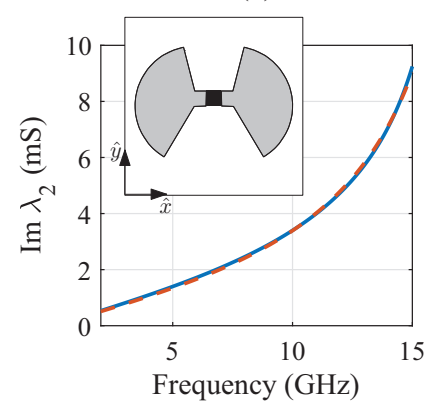

(d)
Fig. 9. Admittances of the equivalent circuit of the unit-cell proposed in [25, Fig. 3] simulated with HFSS (continuous lines) and obtained with the approximation of (18) (dashed lines). (a) and (c) $\lambda_{1}$. (b) and (d) $\lambda_{2}$.

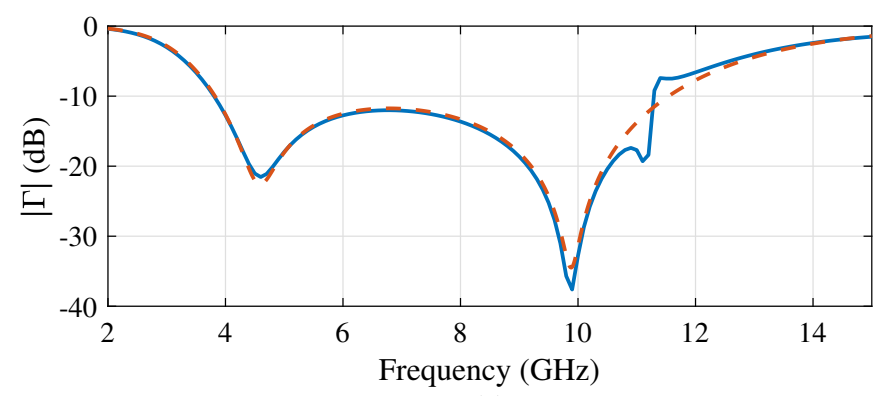

(a)

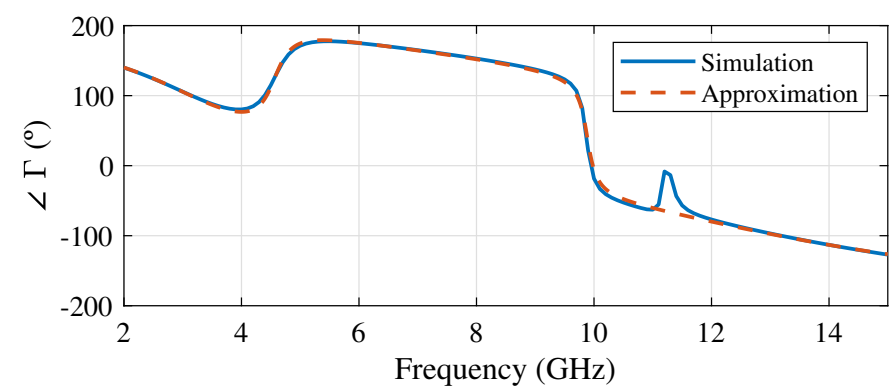

(b)

Fig. 10. Reflection coefficient of the full structure proposed in [25, Fig. 3] simulated with HFSS (continuous lines) and obtained with the approximation of (18) (dashed lines). (a) Magnitude. (b) Phase.

circuit. The agreement between results is excellent, apart from the aforementioned spike at $11.2 \mathrm{GHz}$.

\section{Scatterer With 90 Degrees Rotational Symmetry}

In search of special properties, some authors have resorted to scatterers with 90 degrees rotational symmetry. This is the

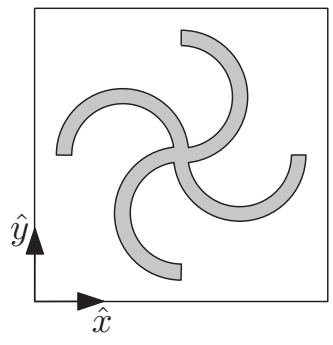

(a)

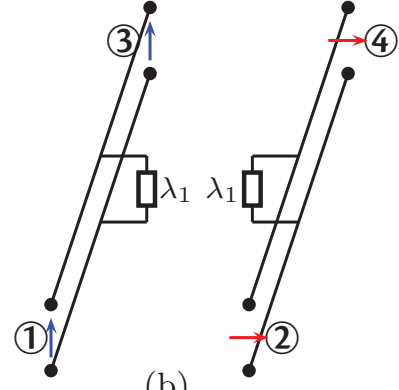

(b)
Fig. 11. (a) Bi-periodic scatterer with 90 degrees rotational symmetry. (b) Equivalent circuit

case of Swastika-like [1], [2], [8] or multi-semicircle-based scatterers [3]. By far the most common case of this rotational symmetry, and also the simplest, is that of a unit cell with two planes of symmetry: simple, Jerusalem, and elaborate crosses [7], [9]-[11], circular and square patches and loops [4], [5], [12], [14], and even more complex geometries [6], [15].

For all these unit cells $S_{11}=S_{22}$ and $S_{12}=0$. Therefore, the results of (8) and (7) are mathematically indeterminate, which corresponds to the fact that any pair of linear polarizations perpendicular to each other can be considered as eigenexcitations of this type of scatterers.

The admittances of the equivalent circuit are equal to each other, and their value is obtained from (11) as

$$
\lambda_{1}=\lambda_{2}=\left(\frac{-2}{\eta_{0}}\right) \frac{S_{11}}{S_{11}+1}
$$

Similarly, the admittance parameters of (3) are particularized to the values

$$
\begin{aligned}
y_{11}=y_{22} & =\lambda_{1}=\lambda_{2} \\
y_{12} & =0
\end{aligned}
$$

Which means that the equivalent circuit is simplified to the one shown in Fig. 11(b). Therefore, no transformers are needed, and the indeterminacy of (8) and (7) is irrelevant.

As an example, the four-semicircle unit cell proposed in [3, Fig. 1(a)], and reproduced in Fig. 11(a), has been analyzed with parameters $r=3, t=1, g=1 \mathrm{~mm}$. After de-embedding the dielectric substrate, the $S$-parameters of the four-port representing the scatterer have been obtained. It was verified that, except for the errors inherent to the numerical simulation, $S_{11}=S_{22}$ and $S_{12}=0$. The equivalent circuit admittances are shown in Fig. 12, admittances that are easily modeled by two LC series resonators in parallel.

\section{Lossless Scatterers}

No matter what symmetry the scatterer exhibits, or the lack of it, when there are no losses there will always be two eigenexcitations in the form of linear polarizations perpendicular to each other. To verify this we start from the fact that, when there are no losses, the unitarity property of the $S$-matrix in (1) leads to

$$
\begin{gathered}
S_{12}\left(1+S_{11}\right)^{*}+S_{22} S_{12}^{*}+S_{12} S_{11}^{*}+\left(1+S_{22}\right) S_{12}^{*}=0 \\
\Rightarrow \operatorname{Re}\left(S_{12}\right)+S_{12} S_{11}^{*}+S_{22} S_{12}^{*}=0
\end{gathered}
$$




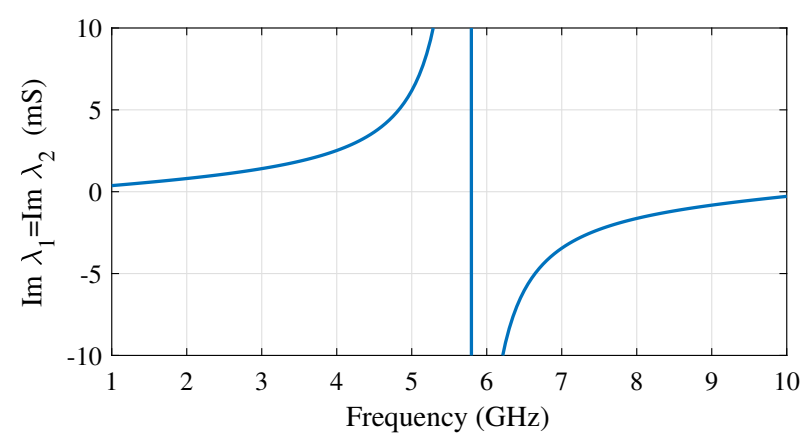

Fig. 12. Admittances of the equivalent circuit for the four-semicircle scatterer proposed in [3], with parameters $r=3, t=1, g=1 \mathrm{~mm}$.

By subtracting from this expression its conjugate

$$
\begin{aligned}
& S_{12}\left(S_{11}^{*}-S_{22}^{*}\right)+S_{12}^{*}\left(S_{22}-S_{11}\right)=0 . \\
& \Rightarrow \quad S_{12}\left(S_{22}-S_{11}\right)^{*}=S_{12}^{*}\left(S_{22}-S_{11}\right)
\end{aligned}
$$

Hence, $S_{12}\left(S_{22}-S_{11}\right) *$ is real. The ratio $2 S_{12} /\left(S_{22}-S_{11}\right)$ will also be real and, in view of (8), so will $\phi$. Consequently, the fields described in (12) and (14) are linear polarizations that constitute the eigenexcitations of the lossless scatterer.

The fact that the lack of losses implies that $\phi$ is real can also be understood from the point of view of the equivalent circuit. As shown in [22] and [23], when the equivalent circuit based on eigenstates represents a lossless structure the transformers are of real turns ratios, which, in the circuit of Fig. 3, implies that $\phi$ is real.

An example of a lossless unit cell without any symmetry is the asymmetrically loaded magnetic dipole presented in [31, Fig. 8(a)]. The extraction of the equivalent circuit proposed in this paper for this dipole leads to an angle $\phi$ that varies slowly with frequency, and to the two admittances $\lambda_{1}$ and $\lambda_{2}$. The admittance $\lambda_{1}$ is negligible, and irrelevant to obtain results using the equivalent circuit. The reactance contributed by $\lambda_{2}$ (the imaginary part of $1 / \lambda_{2}$ ) is shown in Fig. 13. The figure also shows some simple approximations to these parameters: a constant value for $\phi$, and a parallel LC resonator for the reactance. The numerical values of the capacitance and inductance of the resonator are shown at the figure caption. The transformer, with a fixed 4.7:1 turns ratio, comes from the constant angle $\widehat{\phi}=78^{\circ}$ with which $\phi$ is approached.

The equivalent circuit with these simple approximations succeeds in producing a good agreement with the simulated transmission parameters of the unit cell, as shown in Fig. 14. The interest in this case is focused on the transmission when it is the horizontal polarization which impinges in the scatterer. The co-polar transmission will correspond to $1+S_{22}$ (the parameter $S_{24}$ according to (1)), while the counter-polar transmission corresponds to parameter $S_{21}\left(S_{23}\right)$.

\section{E. Lossy Scatterers Without Symmetry Planes}

The scatterers included in the unit cells can be lossy, because they are composed of resistive paints, because they include lumped resistors, or simply because the losses in their conductors are significant.

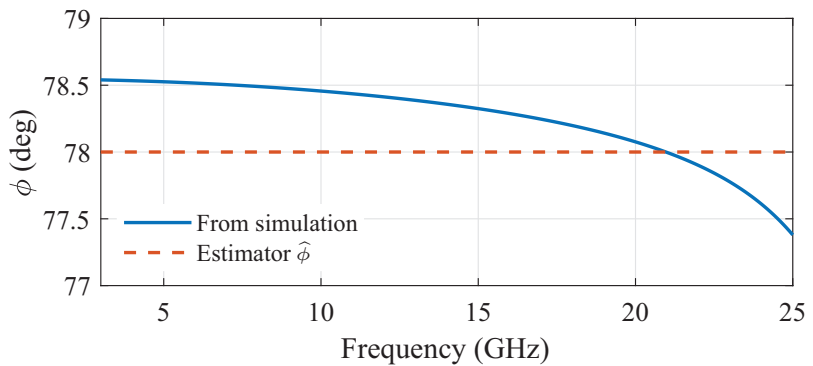

(a)

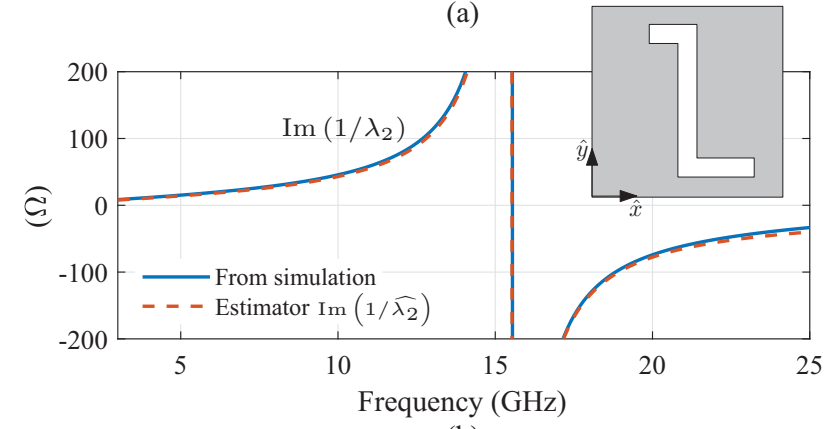

(b)

Fig. 13. Loaded magnetic dipole presented in [31, Fig. 8(a)]. (a) Angle $\phi$ and simple approach $\widehat{\phi}=78^{\circ}$. (b) Reactance of the equivalent circuit and simple approach: $\widehat{\lambda_{2}}=j \omega C+1 /(j \omega L)$, with $L=400 \mathrm{pH}$ and $C=260 \mathrm{fF}$.

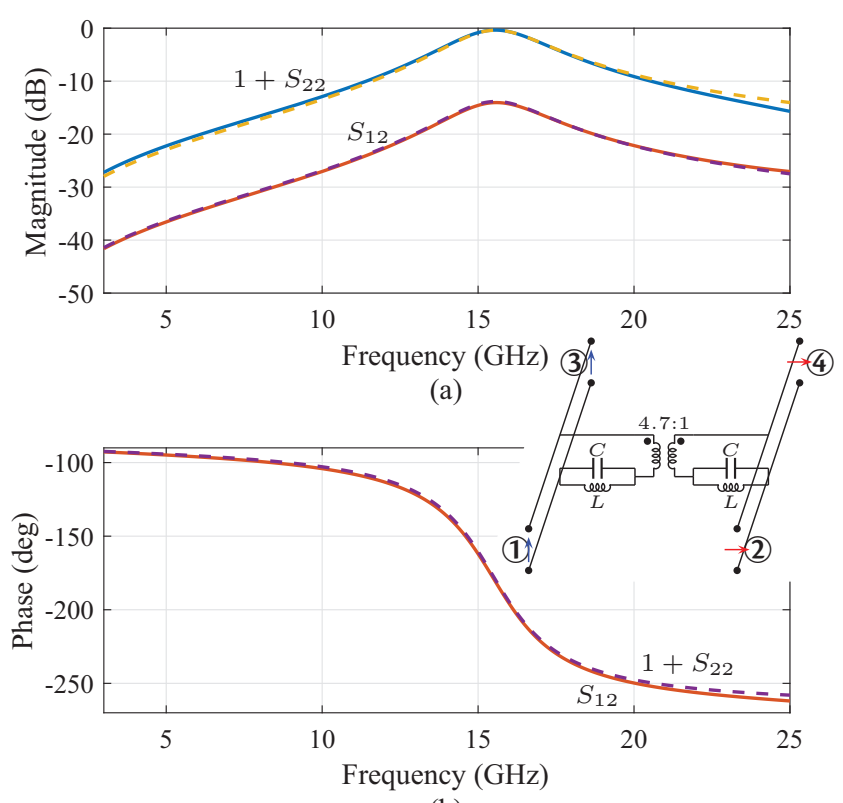

(b)

Fig. 14. Magnitude (a) and phase (b) of the transmission parameters of the loaded magnetic dipole presented in [31, Fig. 8(a)], simulated with HFSS (continuous lines) and obtained with the proposed equivalent circuit (dashed lines).

Even if they are lossy, when they have any of the symmetry properties discussed in the previous sections, the expression (8) leads to real values of $\phi$ and to eigenexcitations in the form of linear polarizations. However, when there are losses 


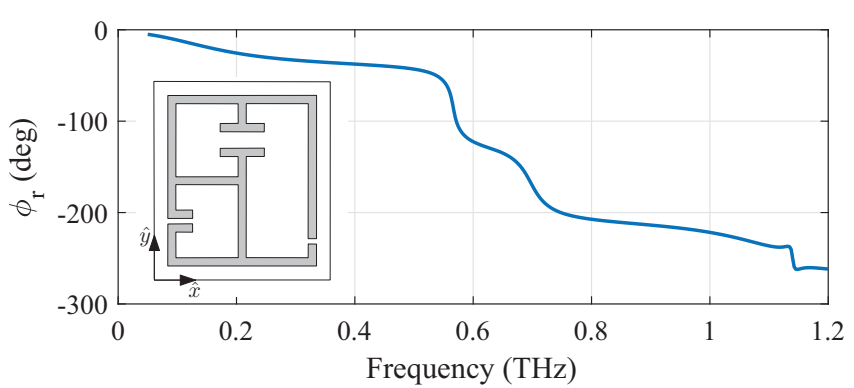

(a)

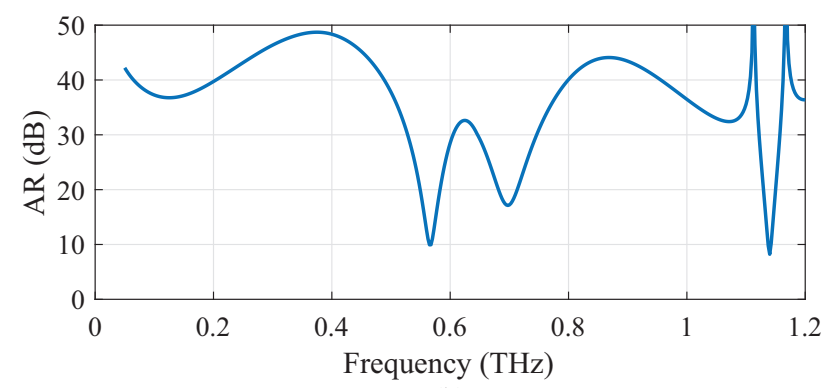

(b)

Fig. 15. (a) Location of the major axes, $\phi_{r}$, and (b) Axial Ratio (in dB) of the eigenexcitations of the multiband frequency-selective surface presented in [32] obtained by simulation with HFSS.

and no symmetry the value obtained for $\phi$ in (8) is complex: $\phi=\phi_{r}+j \phi_{i}$. This situation is not taken into account, or at least not made explicit, in [21]. There, it is stated that the parameters $m$ and $\varphi^{r o t}$ of the expressions [21, (22) and (23)] are real, despite the fact that the elements of the $S$-parameter matrix $\left(\Gamma_{x x}, \Gamma_{y x}\right.$ and $\Gamma_{y y}$ in [21]) are, in general, complex.

For lossy scatterers without any symmetry, the eigenexcitations are no longer linear polarizations, but elliptical ones. These elliptical polarizations (see Appendix) will be characterized by the location of their major axes by means of $\phi_{r}$, their axial ratios $|r|=\left|\operatorname{coth} \phi_{i}\right|$, and their handedness, determined by the sign of $r$.

An example of this kind of unit cell is present in [32]. The scatterer is in this case an intricate aluminum layout over a silicon substrate (see the inset in Fig. 15(a)), whose shape and dimensions are given in [32, Fig. 1] and along that reference's text. The silicon substrate is thick enough so that the simulation of the unit cell by HFSS can be done considering vacuum at the physical input port and silicon at the output port. A simple change of reference impedance at the output electrical ports (3) and (4)) allows the $S$-parameters of the four-port of Fig. 2(a) to be obtained. In the electromagnetic simulations, the thickness of the aluminum strips has been taken into account for the computation of losses. But this thickness is small enough to be neglected as far as the equivalent circuit is concerned, since it has been found that the obtained S-parameters satisfy the relations made explicit in (1).

This bi-periodic surface is designed to operate in the terahertz frequency range, where losses in the aluminum are appreciable enough. As a result, the angle $\phi$ is complex, and

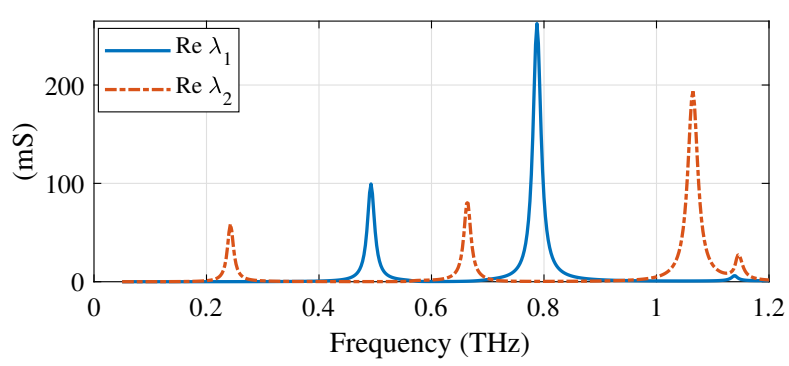

(a)

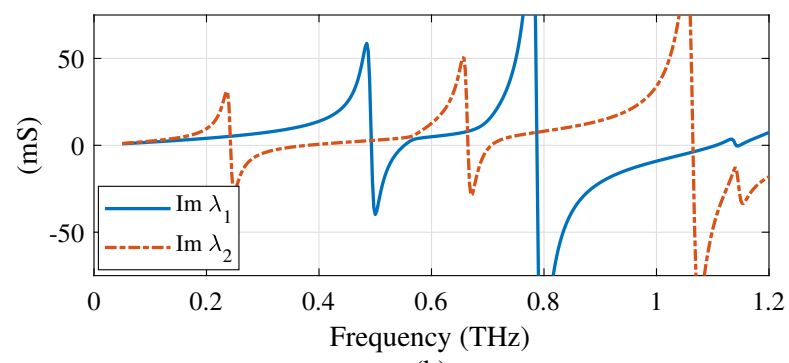

(b)

Fig. 16. Real (a) and imaginary (b) parts of the admittances of the proposed equivalent circuit for the multiband frequency-selective surface presented in [32] obtained by simulation with HFSS.

its real and imaginary parts are shown in Fig. 15, where the imaginary part is represented as the axial ratio in decibels, i.e., $A R(\mathrm{~dB})=20 \log \left|\operatorname{coth} \phi_{i}\right|$. Note that there are frequencies at which the axial ratio is below $10 \mathrm{~dB}$, so the polarizations of the scatterer eigenexcitations are far from linear. It is also interesting to see how the directions of the semi-major axes, $\phi_{r}$, vary with frequency as the different parts of the intricate unit-cell layout become relevant.

As in the other cases, the expressions in (11) provide the circuit admittances, which, in this case, have relevant real parts. These admittances are shown in Fig. 16. The five resonances predicted by the authors of [32] are evident, and are split between the two admittances. There even appears to be a sixth resonance at around $1.14 \mathrm{THz}$.

\section{CONCLUSiON}

The use of the equivalent circuit based on eigenstates for the analysis and modeling of bi-periodic structures has shown all its advantages. In the case of unit cells whose scatterer element is small compared to the unit cell, the circuit has been able to obtain the element orientation and admittances invariant with the angular position in a simple and explicit way. Consequently, it is able to obtain its response in any of its orientations. When the scatterer element is of similar size to the cell it occupies, it is only necessary to model the variation of the position of its eigenexcitations with the element orientation to obtain a similar result. In any case, the circuit reduces the number of elements compared to a network with the usual $\Pi$ or $\mathrm{T}$ topologies, as it contains only two immittances. These are all advantages shared with the approach in [21]. 
Moreover, and unlike [21], the proposed circuit and its decomposition into eigenstates have been able to deal with lossy structures without any further difficulties. The result is a circuit with two immittances that have positive real parts. The modeling of these admittances, in the cases considered so far, has always been feasible with constant and positive RLC elements. This has considerably simplified the handling of unit cells with lossy elements, such as those found in rasorbers.

A number of examples have been shown of how the circuit is simplified and configured according to the symmetry properties of the unit cell it represents. The characteristics of these symmetries are directly transferred and made explicit in the final appearance of the circuit.

It should also be noted that, in the cases of lossy and nonsymmetrical scatterer elements, the circuit contains transformers with complex turns ratios. But the circuit is then able to provide some insight into the occurrence of the complex transformers, which in the end reflects the different character of the unit-cell eigenexcitations. These cease to be linear polarizations and it is the circuit deduction procedure itself that allows us to discover that they become non-orthogonalin-power elliptic polarizations.

\section{APPENDIX}

When $\phi$ is complex, so are the eigenvectors (9). Writing $\phi$ in terms of its real and imaginary parts, $\phi=\phi_{r}+j \phi_{i}$, the eigenvectors are:

$$
\begin{aligned}
& \vec{v}_{1}=\left[\begin{array}{c}
\sin \phi_{r} \cosh \phi_{i}+j \cos \phi_{r} \sinh \phi_{i} \\
\cos \phi_{r} \cosh \phi_{i}-j \sin \phi_{r} \sinh \phi_{i}
\end{array}\right] \\
& \vec{v}_{2}=\left[\begin{array}{c}
-\cos \phi_{r} \cosh \phi_{i}+j \sin \phi_{r} \sinh \phi_{i} \\
\sin \phi_{r} \cosh \phi_{i}+j \cos \phi_{r} \sinh \phi_{i}
\end{array}\right]
\end{aligned}
$$

Let us define two new (real and unit) vectors that correspond to the expressions (9) when $\phi$ is replaced by its real part:

$$
\hat{u}_{1}=\left[\begin{array}{c}
\sin \phi_{r} \\
\cos \phi_{r}
\end{array}\right] \quad ; \quad \hat{u}_{2}=\left[\begin{array}{c}
-\cos \phi_{r} \\
\sin \phi_{r}
\end{array}\right] .
$$

With these new vectors, the eigenvectors can be expressed as

$$
\begin{aligned}
& \vec{v}_{1}=\hat{u}_{1} \cosh \phi_{i}-j \hat{u}_{2} \sinh \phi_{i} \\
& \vec{v}_{2}=j \hat{u}_{1} \sinh \phi_{i}+\hat{u}_{2} \cosh \phi_{i} .
\end{aligned}
$$

For convenience, a parameter $r$ can be defined as $r=\operatorname{coth} \phi_{i}$. Then, vectors $\vec{v}_{1}$ and $\vec{v}_{2}$ can be renormalized to obtain unit eigenvectors in the case of complex $\phi$ :

$$
\hat{v}_{1}=\frac{r \hat{u}_{1}-j \hat{u}_{2}}{\sqrt{1+r^{2}}} \quad ; \quad \hat{v}_{2}=\frac{j \hat{u}_{1}+r \hat{u}_{2}}{\sqrt{1+r^{2}}} .
$$

These two vectors represent two elliptical polarizations with the same axial ratio, $|r|$, with the same handedness (sign of $r$ ) and whose semi-major axes are perpendicular to each other. While one of them will have its semi-major axis in the $\hat{u}_{1}$ direction, the other will have it in the $\hat{u}_{2}$ direction, as shown in Fig. 17.

Had these elliptic polarizations a different handedness, they would be orthogonal. But, sharing the same handedness, they do not obey the power orthogonality relation as the linear polarizations do. This fact runs parallel to the fact that the

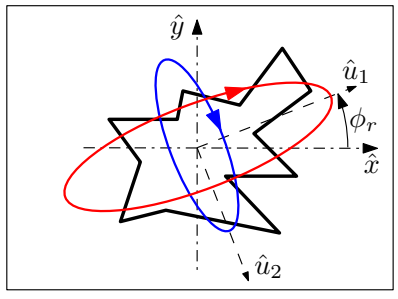

Fig. 17. Unit cell of a lossy scatterer without symmetries. Location of the two elliptically polarized eigenexcitations.

excitations of the proposed equivalent circuit are not power orthogonal when the transformer turns ratios are not real [23].

Note that any arbitrary field always admits a decomposition into these two eigenexcitations. For instance, an electric field $\vec{E}$ can be written as:

$$
\vec{E}=c_{1} \hat{v}_{1}+c_{2} \hat{v}_{2}
$$

where

$$
\begin{aligned}
& c_{1}=\frac{\sqrt{r^{2}+1}}{r^{2}-1}\left(r \vec{E} \cdot \hat{u}_{1}-j \vec{E} \cdot \hat{u}_{2}\right) \\
& c_{2}=\frac{\sqrt{r^{2}+1}}{r^{2}-1}\left(j \vec{E} \cdot \hat{u}_{1}+r \vec{E} \cdot \hat{u}_{2}\right) .
\end{aligned}
$$

In view of the expressions of these two coefficients, the decomposition into eigenexcitations will not be possible when the axial ratio $|r|$ is very close to unity, since both coefficients would tend to infinity. But this cannot happen. From (8) it follows that

$$
\phi_{i}=\frac{1}{2} \arg \left(\frac{j\left(S_{22}-S_{11}\right)-2 S_{12}}{j\left(S_{22}-S_{11}\right)+2 S_{12}}\right) .
$$

Therefore, $\left|\phi_{i}\right| \leq \pi / 2$ and the axial ratio has as lower bound: $|r| \geq \operatorname{coth}(\pi / 2)=1.09$.

\section{REFERENCES}

[1] R. Natarajan, M. Kanagasabai, S. Baisakhiya, R. Sivasamy, S. Palaniswamy, and J. K. Pakkathillam, "A compact frequency selective surface with stable response for WLAN applications," IEEE Antennas Wireless Propag. Lett., vol. 12, pp. 718-720, 2013.

[2] S. Narayan, G. Gulati, B. Sangeetha, and R. U. Nair, "Novel metamaterial-element-based FSS for airborne radome applications," IEEE Trans. Antennas Propag., vol. 66, no. 9, pp. 4695-4707, Sep. 2018.

[3] D. Ferreira, I. Cuinas, T. R. Fernandes, and R. F. Caldeirinha, "Multisemicircle-based single- and dual-band frequency-selective surfaces: Achieving narrower bandwidth and improved oblique incidence angular stability," IEEE Antennas Propag. Mag., vol. 61, no. 2, pp. 32-39, April 2019.

[4] W. Y. Yong, S. K. Abdul Rahim, M. Himdi, F. C. Seman, D. L. Suong, M. R. Ramli, and H. A. Elmobarak, "Flexible convoluted ring shaped FSS for X-band screening application," IEEE Access, vol. 6, pp. $11657-$ $11665,2018$.

[5] G.-B. Wu, S.-W. Qu, Y.-X. Wang, and S. Yang, "Nonuniform FSSbacked reflectarray with synthesized phase and amplitude distribution," IEEE Trans. Antennas Propag., vol. 66, no. 12, pp. 6883-6892, Dec 2018.

[6] X.-J. Sheng, J.-J. Fan, N. Liu, and C.-B. Zhang, "A miniaturized dual-band FSS with controllable frequency resonances," IEEE Microw. Wireless Compon. Lett., vol. 27, no. 10, pp. 915-917, Oct 2017.

[7] Y. Chen, L. Chen, H. Wang, X.-T. Gu, and X.-W. Shi, "Dual-band crossed-dipole reflectarray with dual-band frequency selective surface," IEEE Antennas Wireless Propag. Lett., vol. 12, pp. 1157-1160, 2013.

[8] T. Hong, W. Xing, Q. Zhao, Y. Gu, and S. Gong, "Single-layer frequency selective surface with angular stability property," IEEE Antennas Wireless Propag. Lett., vol. 17, no. 4, pp. 547-550, April 2018. 
[9] E. Arnieri, F. Greco, and G. Amendola, "A broadband, wide-angle scanning, linear-to-circular polarization converter based on standard Jerusalem cross frequency selective surfaces," IEEE Trans. Antennas Propag., vol. 69, no. 1, pp. 578-583, Jan 2021.

[10] V. Krushna Kanth and S. Raghavan, "EM design and analysis of frequency selective surface based on substrate-integrated waveguide technology for airborne radome application," IEEE Trans. Microw. Theory Techn., vol. 67, no. 5, pp. 1727-1739, May 2019.

[11] D. Kundu, S. Baghel, A. Mohan, and A. Chakrabarty, "Design and analysis of printed lossy capacitive surface-based ultrawideband lowprofile absorber," IEEE Trans. Antennas Propag., vol. 67, no. 5, pp. 3533-3538, May 2019.

[12] X.-D. Hu, X.-L. Zhou, L.-S. Wu, L. Zhou, and W.-Y. Yin, "A miniaturized dual-band frequency selective surface (FSS) with closed loop and its complementary pattern," IEEE Antennas Wireless Propag. Lett., vol. 8, pp. 1374-1377, 2009.

[13] M. Guo, T. Guo, Q. Cheng, Y. Zheng, and Y. Fu, "Frequency selective rasorber with anisotropic transmission band," IEEE Antennas Wireless Propag. Lett., vol. 20, no. 2, pp. 155-159, Feb 2021.

[14] M. Li, S. Xiao, Y.-Y. Bai, and B.-Z. Wang, "An ultrathin and broadband radar absorber using resistive FSS," IEEE Antennas Wireless Propag. Lett., vol. 11, pp. 748-751, 2012.

[15] S. C. Bakshi, D. Mitra, and F. L. Teixeira, "Wide-angle broadband rasorber for switchable and conformal application," IEEE Trans. Microw. Theory Techn., vol. 69, no. 2, pp. 1205-1216, Feb 2021.

[16] S. Maci, M. Caiazzo, A. Cucini, and M. Casaletti, "A pole-zero matching method for EBG surfaces composed of a dipole FSS printed on a grounded dielectric slab," IEEE Trans. Antennas Propag., vol. 53, no. 1, pp. 70-81, Jan 2005.

[17] F. Costa and A. Monorchio, "Closed-form analysis of reflection losses in microstrip reflectarray antennas," IEEE Trans. Antennas Propag., vol. 60, no. 10 , pp. $4650-4660$, Oct 2012.

[18] F. Mesa, R. Rodriguez-Berral, and F. Medina, "Unlocking complexity using the eca: The equivalent circuit model as an efficient and physically insightful tool for microwave engineering," IEEE Microw. Mag., vol. 19, no. 4, pp. 44-65, June 2018.

[19] C. Molero and M. García-Vigueras, "Circuit modeling of 3-D cells to design versatile full-metal polarizers," IEEE Trans. Microw. Theory Techn., vol. 67, no. 4, pp. 1357-1369, April 2019.

[20] F. Costa, A. Monorchio, and G. Manara, "Efficient analysis of frequencyselective surfaces by a simple equivalent-circuit model," IEEE Antennas Propag. Mag., vol. 54, no. 4, pp. 35-48, Aug 2012.

[21] M. Borgese and F. Costa, "A simple equivalent circuit approach for anisotropic frequency-selective surfaces and metasurfaces," IEEE Trans. Antennas Propag., vol. 68, no. 10, pp. 7088-7098, Oct 2020.

[22] A. Hernández-Escobar, E. Abdo-Sánchez, P. Mateos-Ruiz, J. Esteban, T. M. Martín-Guerrero, and C. Camacho-Peñalosa, "An equivalentcircuit topology for lossy non-symmetric reciprocal two-ports," IEEE J. Microw., vol. 1, no. 3, pp. 810-820, 2021.

[23] E. Abdo-Sánchez, C. Camacho-Peñalosa, T. M. Martín-Guerrero, and J. Esteban, "Equivalent circuits for nonsymmetric reciprocal two ports based on eigenstate formulation," IEEE Trans. Microw. Theory Techn., vol. 65, no. 12, pp. 4812-4822, Dec. 2017.

[24] D.-U. Sim, J.-H. Kwon, Y.-J. Chong, and S.-O. Park, "Design of electromagnetic wave absorber using periodic structure and method to broaden its bandwidth based on equivalent circuit-based analysis," IET Microw., Antennas and Propag., vol. 9, pp. 142-150(8), January 2015.

[25] X. Q. Lin, P. Mei, P. C. Zhang, Z. Z. D. Chen, and Y. Fan, "Development of a resistor-loaded ultrawideband absorber with antenna reciprocity," IEEE Trans. Antennas Propag., vol. 64, no. 11, pp. 4910-4913, Nov 2016.

[26] M. Guo, Q. Chen, T. Bai, K. Wei, and Y. Fu, "Wide transmission band frequency-selective rasorber based on convoluted resonator," IEEE Antennas Wireless Propag. Lett., vol. 19, no. 5, pp. 846-850, May 2020.

[27] Y. Jia, A. Wu, Y. Liu, W. Zhang, and Z. Zhou, "Dual-polarization frequency-selective rasorber with independently controlled dual-band transmission response," IEEE Antennas Wireless Propag. Lett., vol. 19, no. 5, pp. 831-835, May 2020

[28] Z. Yang, W. Jiang, Q. Huang, and T. Hong, "A 2.5-D miniaturized frequency-selective rasorber with a wide high-transmission passband," IEEE Antennas Wireless Propag. Lett., vol. 20, no. 7, pp. 1140-1144, July 2021

[29] G. Pérez-Palomino and J. E. Page, "Bimode Foster's equivalent circuit of arbitrary planar periodic structures and its application to design polarization controller devices," IEEE Trans. Antennas Propag., vol. 68 no. 7, pp. 5308-5321, 2020.
[30] M. Rotaru and J. Sykulski, "Numerical investigation of Fano resonances in metamaterials with electric asymmetry," IET Sci., Meas. \& Technol. vol. 6, pp. 386-393(7), September 2012.

[31] C. Molero, A. Alex-Amor, F. Mesa, Á. Palomares-Caballero, and P. Padilla, "Cross-polarization control in FSSs by means of an equivalent circuit approach," IEEE Access, vol. 9, pp. 99513-99 525, 2021.

[32] S. Qiao, Y. Zhang, Y. Zhao, Y. Zhou, S. Liang, and Z. Yang, "Multiband frequency-selective surface with five resonance peaks in terahertz band," IEEE Trans. THz Sci. Technol., vol. 6, no. 2, pp. 292-299, March 2016. 